OPEN ACCESS

Edited by: Andreas Nord

Lund University, Sweden

Reviewed by:

Sylvain Giroud,

University of Veterinary Medicine

Vienna, Austria

Simon Charles Griffith

Macquarie University, Australia

${ }^{*}$ Correspondence:

R. Mark Brigham

mark.brigham@uregina.ca

Specialty section:

This article was submitted to

Ecophysiology,

a section of the journal

Frontiers in Ecology and Evolution

Received: 04 September 2021

Accepted: 16 December 2021

Published: 10 January 2022

Citation:

Czenze ZJ, Myers M, Collins D and Brigham RM (2022) Captive Tawny Frogmouths Exhibit Similar Body Temperature Patterns as Wild

Individuals.

Front. Ecol. Evol. 9:770788. doi: $10.3389 /$ fevo.2021.770788

\section{Captive Tawny Frogmouths Exhibit Similar Body Temperature Patterns as Wild Individuals}

\author{
Zenon J. Czenze ${ }^{1}$, Mark Myers ${ }^{2}$, Darin Collins ${ }^{2}$ and R. Mark Brigham ${ }^{3 *}$ \\ ${ }^{1}$ Centre for Behavioural and Physiological Ecology, Zoology, University of New England, Armidale, NSW, Australia, \\ ${ }^{2}$ Woodland Park Zoo, Seattle, WA, United States, ${ }^{3}$ Department of Biology, University of Regina, Regina, SK, Canada
}

Free-ranging tawny frogmouths (Podargus strigoides) typically defend body temperature $\left(T_{b}\right)$ between 38 and $40^{\circ} \mathrm{C}$ during activity and allow it to fall to $29^{\circ} \mathrm{C}$ during cold evenings. However, this pattern of nightly $T_{b}$ decline has not been elicited in captivity during shortterm respirometry measurements. We used implanted $T_{b}$ loggers to record the $T_{b}$ of two captive tawny frogmouths from 24 September to 24 December 2019 to determine if the conditions in captivity would elicit similar $T_{b}$ patterns to those measured in the wild. We recorded an average $T_{b}$ of $34.8 \pm 1.1$ and $35.6 \pm 1.0^{\circ} \mathrm{C}$ for the two birds and minimum $T_{b}$ of 31.0 and $32.0^{\circ} \mathrm{C}$. Minimum daily $T_{b}$ was correlated between the two individuals, and the minimum $T_{b}$ of both individuals was correlated with minimum daily $T_{a}$. Our results highlight the need to keep birds under appropriate captive conditions to perform physiological research that produces results which mirror responses by individuals in the wild.

Keywords: torpor, frogmouths, captive animals, body temperature, metabolism

\section{INTRODUCTION}

Heterothermy and torpor are physiological states characterized by a reduction in metabolic rate and subsequently body temperature ( $T_{b}$; Geiser, 2004). Although once commonly associated with mammals from seasonally cold climates to save energy, it is now apparent that many mammals and birds from subtropical and tropical latitudes also employ heterothermy on a regular basis (Genoud, 1993; Geiser and Brigham, 2012; Geiser, 2020; Reher and Dausmann, 2021). Among birds, members of Apodiformes and Caprimulgiformes readily enter torpor in response to energy or water shortages in the wild (Krüger et al., 1982; Smit et al., 2011; Brigham et al., 2012; Wolf et al., 2020). However, there is limited evidence that captive caprimulgids mirror the thermoregulatory patterns of their wild counterparts although birds in general appear less likely to employ heterothermy under captive conditions compared to mammals (Geiser et al., 2000).

One caprimulgid that has been studied in both captivity and the wild is the tawny frogmouth (Podargus strigoides). In the southern hemisphere, free-ranging tawny frogmouths regularly enter daily torpor during the austral winter (Körtner et al., 2000, 2001). In the wild, tawny frogmouths body temperature $\left(T_{b}\right)$ during activity is typically between 38 and $40^{\circ} \mathrm{C}$, but these birds allow $T_{b}$ to fall to $29^{\circ} \mathrm{C}$ during cold evenings (Körtner et al., 2000). Captive tawny frogmouths regulate their $T_{b}$ near $38^{\circ} \mathrm{C}$; however, there is no direct evidence for the similar drops in $T_{b}$ by captive birds (McNab and Bonaccorso, 1995; Bech and Nicol, 1999). The difference between the 
thermoregulatory patterns of wild and captive individuals is likely due, in part, to the nature of the animal's captivity, duration of measurements, and that most measurements were made in respirometry chambers. Our goal was to determine if the $T_{b}$ of long-term captive tawny frogmouths measured using implanted $T_{b}$ loggers and recorded over months instead of hours would better reflect the patterns that have been observed in the wild. We predicted that under these conditions, the birds would exhibit patterns of heterothermy similar to those that measured in the wild.

\section{MATERIALS AND METHODS}

We undertook this study at the Woodland Park Zoo in Seattle, Washington $\left(47.6682^{\circ} \mathrm{N}, 122.3508^{\circ} \mathrm{W}\right)$ from 24 September to 24 December 2019. Both birds (a male and a female) used in this study were hatched in captivity. The male hatched 20 April 1998 at the Chester Zoo, Chester, United Kingdom and the female hatched 11 July 2010 at the Woodland Park Zoo. Birds were housed in an off-exhibit indoor-outdoor enclosure. The indoor portion measured $2.3 \times 2.3 \times 2.4 \mathrm{~m}$, where temperature was controlled at $18-22^{\circ} \mathrm{C}$, and an outdoor portion measuring $6.1 \times 2.9 \times 2.9 \mathrm{~m}$. Birds had access to both sections at all times. Birds were fed with Zophobas larvae, adult crickets, and mice ad libitum twice each day. The indoor portion of the enclosure was cleaned daily.

Body temperature was recorded hourly using iButtons (DS1922L, $\sim 3 \mathrm{~g}$, range -40 to $85^{\circ} \mathrm{C}$, accuracy $0.5^{\circ} \mathrm{C}$; Maxim Integrated Products, Inc., Sunnyvale, CA, United States) calibrated by the manufacturer and implanted on 19 September by a registered veterinarian. The male weighed $556 \mathrm{~g}$ and the female $464 \mathrm{~g}$. The iButtons were programmed to begin recording on 1 October, 11 days after surgery. iButtons were previously sterilized with an ethylene oxide gas (Anprolene, Andersen Sterilizers, Inc., Haw River, NC, United States) and covered with an inert bone wax (Ethicon Bone Wax 2.5 g; Medline, Northfield, IL, United States), and were implanted intracoelomically caudal to the liver and adjacent to the right wall of the coelomic cavity between the caudal air sacs. Each bird was mask induced with isoflurane (Foranet, Baxter Healthcare Corporation, Deerfield, IL, United States) and maintained on isoflurane mixed with $100 \%$ oxygen and received meloxicam (0.5 mg I.M.; $1 \mathrm{mg} / \mathrm{kg}$ ) (Boehringer Ingelhiem, St. Joseph, MOUSA), and a bolus of lactated ringer's solution ( $5 \mathrm{ml} \mathrm{S.C.;} 15 \mathrm{mg} / \mathrm{kg}$ ) (McGaw, Irvine, CA, United States) was injected once induction was completed.

To examine the similarity between the $T_{b}$ of both frogmouths we fit a linear model to the minimum daily $T_{b}$ of frogmouth 1 to minimum daily $T_{b}$ of frogmouth 2 . To investigate the influence of ambient temperature on body temperature, we fit a linear model to the minimum daily $T_{b}$ of frogmouths to minimum daily $T_{a}$. All analyses were done in R 3.6.3 (R Core Team, 2019).

\section{RESULTS}

From 1 October to 24 December 2019 we recorded a total of 171 bird days of $T_{b}$ data for the 2 individuals. During the study period, the average ambient temperature $\left(T_{a}\right)$ was $11.9 \pm 6.3^{\circ} \mathrm{C}$, maximum $T_{a}=30.3^{\circ} \mathrm{C}$, minimum $T_{a}=-4.1^{\circ} \mathrm{C}$, maximum daily $T_{a}$ amplitude $=15.3^{\circ} \mathrm{C}$ (Figure 1). Comparatively, we recorded an average $T_{b}$ of $34.8 \pm 1.1$ and $35.6 \pm 1.0^{\circ} \mathrm{C}$ for the two birds, maximum $T_{b}$ of 38.0 and $37.0^{\circ} \mathrm{C}$, minimum $T_{b}$ of 31.0 and $32.0^{\circ} \mathrm{C}$, and both birds exhibited a maximum daily amplitude in $T_{b}$ of $4.5^{\circ} \mathrm{C}$ on a day when the corresponding $T_{a}$ amplitude was $13.2^{\circ} \mathrm{C}$.

Minimum daily $T_{b}$ was correlated between the two individuals $\left(F=805.3_{1,78}, t=28.4, p<0.001, R^{2}=0.91\right.$; Figure 2A), and the minimum $T_{b}$ of both individuals was correlated with minimum daily $T_{a}\left(F=805.3_{1,158}, t=5.5, p<0.001, R^{2}=0.16\right.$; Figure 2B). Even during cold weather and snowfall events both individuals preferred to remain in open enclosure exposed to ambient temperature and conditions (Figure 3).

\section{DISCUSSION}

We recorded $T_{b}$ patterns by captive tawny frogmouths that were similar to those recorded for birds in the wild. The maximum $\left(38.0^{\circ} \mathrm{C}\right)$ and minimum $\left(31.0^{\circ} \mathrm{C}\right) \mathrm{T}_{b}$ we measured were similar to those values for free ranging individuals (Körtner et al., 2000). However, captive individuals defended an average $T_{b}$ that was nearly $4^{\circ} \mathrm{C}$ lower than their wild counterparts. This may be due, in part, to the colder temperatures to which the zoo birds were exposed, or a lack of activity comparable to that of their wild counterparts. Körtner et al. (2000) found that dawn episodes of heterothermy were shorter $(3.5 \pm 1.2 \mathrm{~h})$ than at night $(7.0 \pm 1.2 \mathrm{~h})$ and often terminated with passive re-warming in the sun. We recorded similar patterns as $T_{b}$ reductions were all associated with night, and the subsequent $T_{b}$ increases with dawn.

We found that $T_{b}$ patterns of both birds were highly consistent and overnight $T_{b}$ was within $1^{\circ} \mathrm{C}$ of each other over the 88day period that we had overlapping data. Further, both birds exhibited greater drops in $T_{b}$ during colder evenings. However, minimum $T_{b}$ for both birds ranged from only $31-36^{\circ} \mathrm{C}$, while minimum $T_{a}$ over this period ranged from -4.1 to $11.9^{\circ} \mathrm{C}$ suggesting that they may defend a minimum set-point $T_{b}$. Nonetheless, as these birds were fed ad libitum, it appears that $T_{b}$ reductions in these two individuals were influenced by $T_{a}$. Although their captive and wild diets are similar (i.e., insects and small vertebrates) the possibility remains that the differences in $T_{b}$ we recorded were influenced by diet. Although they have access to ad libitum food, captive frogmouths lose mass over winter (Stulberg et al., 2018) in a manner similar to their wild counterparts despite the energy savings likely due to the use of heterothermy and the relative lack of activity compared to wild birds.

Here we provide re-assuring evidence that captive caprimulgids will exhibit similar physiological responses compared to free-ranging birds given the appropriate conditions. One factor that appears to be important, based on our results and in contrast to short term recordings from respirometry chambers, is long-term stable captivity and access to natural conditions. We stress that although birds had free 


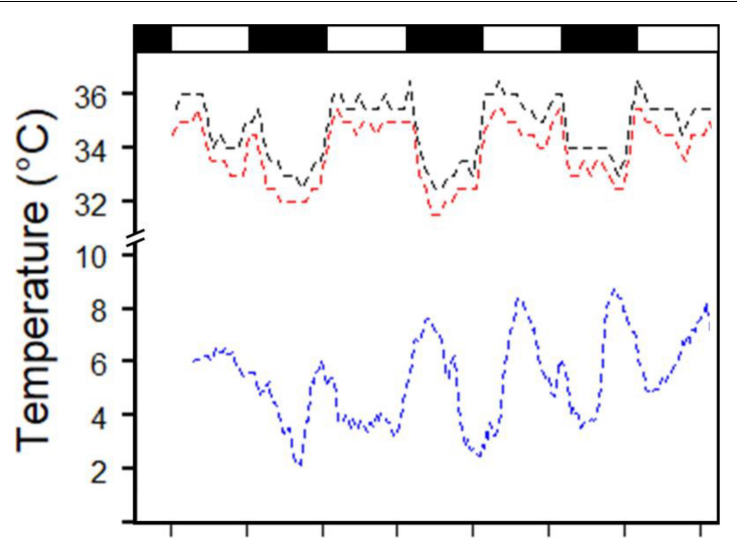

FIGURE 1 | Fluctuations in body temperature of two captive tawny frogmouths (Podargus strigoides) from Woodland Park Zoo in Seattle, Washington (red and black dashed lines, measured by an internal transmitter) and in air temperature (blue dashed lines solid line) over 4 days in December. Note the break in $y$-axis. Black rectangles represent night.

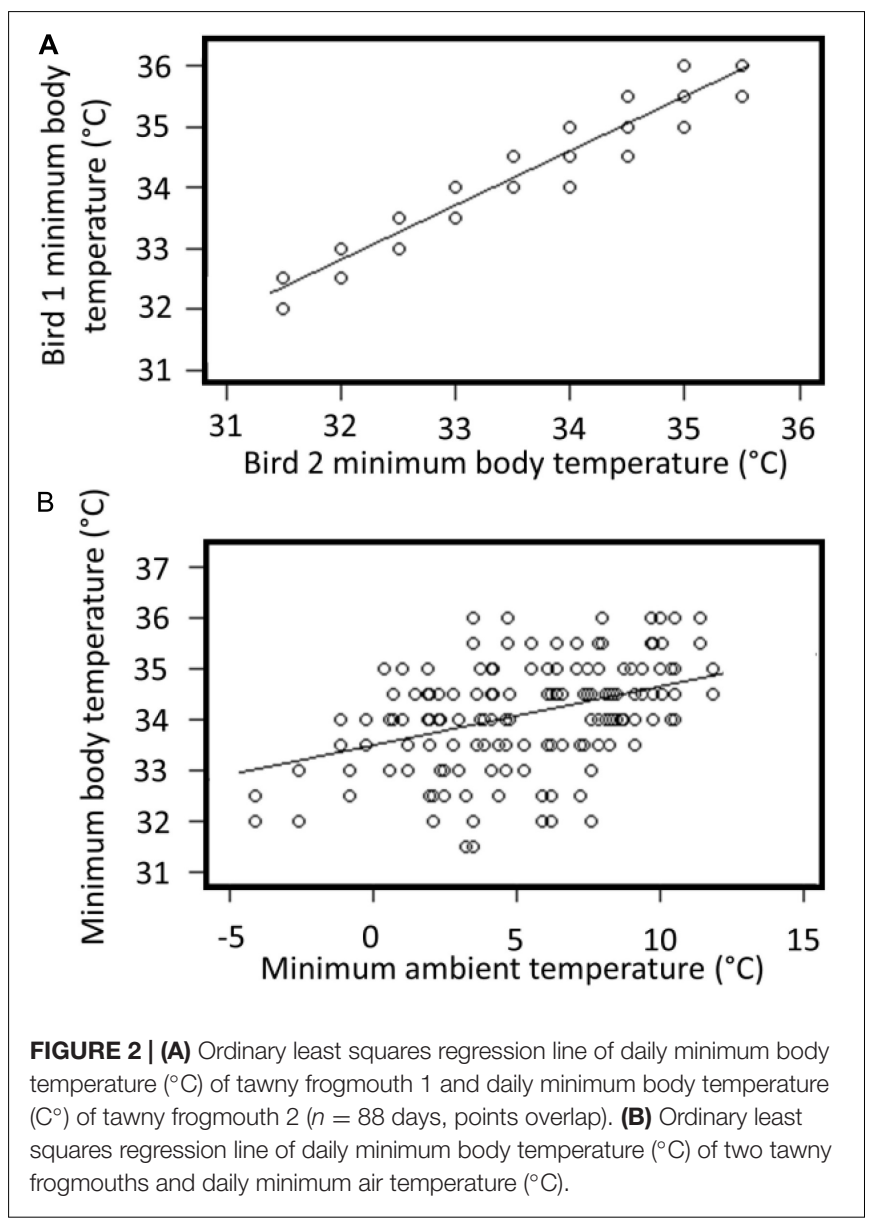

access to heated enclosures, during even cold weather and snowfall events they remained in the exposed section of the enclosure. Taken together, our results suggest that under appropriate captive conditions, physiological research that

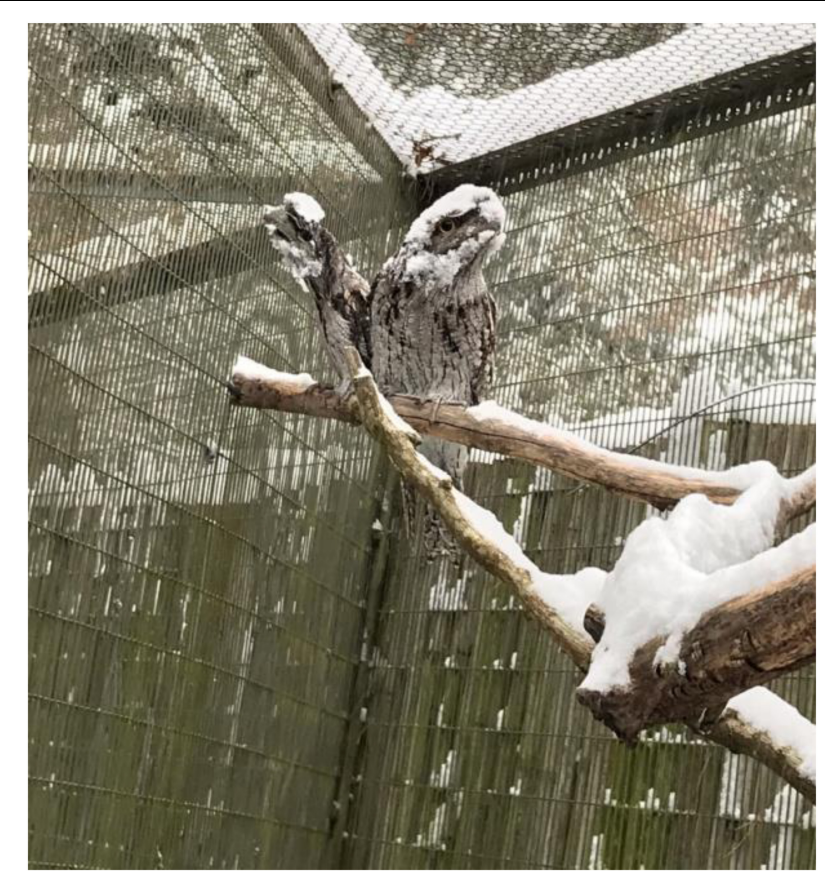

FIGURE 3 | Two captive tawny frogmouths (Podargus strigoides) from Woodland Park Zoo in Seattle, Washington after a snowfall event.

produces results which mirror responses by birds in the wild is possible.

\section{DATA AVAILABILITY STATEMENT}

The original contributions presented in the study are included in the article/supplementary material, further inquiries can be directed to the corresponding author.

\section{ETHICS STATEMENT}

The animal study was reviewed and approved by Presidents Committee on Animal Care, University of Regina. Woodland Park Zoo also approved the protocol.

\section{AUTHOR CONTRIBUTIONS}

RMB and ZJC developed the idea and co-wrote the draft manuscript. MM was involved in project development and animal husbandry and edited the manuscript. DC conducted surgical implants and edited the manuscript. All authors contributed to the article and approved the submitted version.

\section{ACKNOWLEDGMENTS}

The authors would like to thank Bill McDowell, Sue Andersen, and Stephanie Miller for providing daily oversight and care for the frogmouths involved in this study. 


\section{REFERENCES}

Bech, C., and Nicol, S. C. (1999). Thermoregulation and ventilation in the tawny frogmouth, Podargus strigoides: a low-metabolic avian species. Aust. J. Zool. 47, 143-153. doi: 10.1071/ZO98058

Brigham, R. M., McKechnie, A. E., Doucette, L. I., and Geiser, F. (2012). "Heterothermy in caprimulgid birds: a review of inter- and intraspecific variation in free-ranging populations," in Living in a Seasonal World: Thermoregulatory and Metabolic Adaptations, eds T. Ruf, C. Bieber, W. Arnold, and E. Millesi (Berlin: Springer), 175-187. doi: 10.1007/978-3-642-28678-0_16

Geiser, F. (2004). Metabolic rate and body temperature reduction during hibernation and daily torpor. Annu. Rev. Physiol. 66, 239-274. doi: 10.1146/ annurev.physiol.66.032102.115105

Geiser, F. (2020). Seasonal Expression of avian and mammalian daily torpor and hibernation: not a simple summer-winter Affair†. Front. Physiol. 11:436. doi: 10.3389/fphys.2020.00436

Geiser, F., and Brigham, R. M. (2012). "The other functions of torpor," in Living in a Seasonal World: Thermoregulatory and Metabolic Adaptations, eds T. Ruf, C. Bieber, W. Arnold, and E. Millesi (Berlin: Springer-Verlag), 109-121. doi: 10.1007/978-3-642-28678-0_10

Geiser, F., Holloway, J. C., Körtner, G., Maddocks, T. A., Turbill, C., and Brigham, R. M. (2000). "Do patterns of torpor differ between free-ranging and captive mammals and birds?," in Life in the Cold: Eleventh International Hibernation Symposium, eds G. Heldmaier and M. Klingenspor (Berlin: Springer), 95-102. doi: 10.1007/978-3-662-04162-8_10

Genoud, M. (1993). Temperature regulation in subtropical tree bats. Comp. Biochem. Physiol. Part A Physiol. 104, 321-331. doi: 10.1016/0300-9629(93) 90324-w

Körtner, G., Brigham, R. M., and Geiser, F. (2000). Winter torpor in a large bird. Nature 407, 318. doi: 10.1038/35030297

Körtner, G., Brigham, R. M., and Geiser, F. (2001). Torpor in free-ranging tawny frogmouths (Podargus strigoides). Physiol. Biochem. Zool. 74, 789-797. doi: 10.1086/324097

Krüger, K., Prinzinger, R., and Schuchmann, K. L. (1982). Torpor and metabolism in hummingbirds. Comp. Biochem. Physiol. Part A Physiol. 73, 679-689. doi: 10.1016/0300-9629(82)90275-4
McNab, B. K., and Bonaccorso, F. J. (1995). The energetics of Australasian swifts, frogmoths, and nightjars. Physiol. Zool. 68, 245-261. doi: 10.1086/physzool.68. 2.30166502

R Core Team (2019). R: A Language and Environment for Statistical Computing. Vienna: R Foundation for Statistical Computing.

Reher, S., and Dausmann, K. H. (2021). Tropical bats counter heat by combining torpor with adaptive hyperthermia. Proc. Biol. Sci. 288:20202059. doi: 10.1098/ rspb.2020.2059

Smit, B., Boyles, J. G., Brigham, R. M., and McKechnie, A. E. (2011). Torpor in dark times: patterns of heterothermy are associated with the lunar cycle in a nocturnal bird. J. Biol. Rhythms 26, 241-248. doi: 10.1177/0748730411402632

Stulberg, A., Myers, M., and Brigham, R. M. (2018). Seasonal body mass fluctuations of captive tawny frogmouths (Podargus strigoides) are consistent with seasonal heterothermy. J. Ornithol. 159, 303-306.

Wolf, B. O., McKechnie, A. E., Schmitt, C. J., Czenze, Z. J., Johnson, A. B., and Witt, C. C. (2020). Extreme and variable torpor among high-elevation Andean hummingbird species: Extreme torpor in Andean hummingbirds. Biol. Lett. 16:20200428. doi: 10.1098/rsbl.2020.0428

Conflict of Interest: The authors declare that the research was conducted in the absence of any commercial or financial relationships that could be construed as a potential conflict of interest.

Publisher's Note: All claims expressed in this article are solely those of the authors and do not necessarily represent those of their affiliated organizations, or those of the publisher, the editors and the reviewers. Any product that may be evaluated in this article, or claim that may be made by its manufacturer, is not guaranteed or endorsed by the publisher.

Copyright (c) 2022 Czenze, Myers, Collins and Brigham. This is an open-access article distributed under the terms of the Creative Commons Attribution License (CC BY). The use, distribution or reproduction in other forums is permitted, provided the original author(s) and the copyright owner(s) are credited and that the original publication in this journal is cited, in accordance with accepted academic practice. No use, distribution or reproduction is permitted which does not comply with these terms. 